\title{
A massive galaxy in its core formation phase three billion years after the Big Bang
}

Erica Nelson ${ }^{1}$, Pieter van Dokkum ${ }^{1}$, Marijn Franx ${ }^{2}$, Gabriel Brammer ${ }^{3}$, Ivelina Momcheva ${ }^{1}$, Natascha Förster Schreiber ${ }^{4}$, Elisabete da Cunha ${ }^{5}$, Linda Tacconi ${ }^{4}$, Rachel Bezanson ${ }^{6}$, Allison Kirkpatrick ${ }^{7}$, Joel Leja ${ }^{1}$, Hans-Walter Rix ${ }^{5}$, Rosalind Skelton ${ }^{8}$, Arjen van der $\mathrm{Wel}^{5}$, Katherine Whitaker ${ }^{9} \&$ Stijn Wuyts ${ }^{4}$

Most massive galaxies are thought to have formed their dense stellar cores in early cosmic epochs $s^{1-3}$. Previous studies have found galaxies with high gas velocity dispersions ${ }^{4}$ or small apparent sizes ${ }^{5-7}$, but so far no objects have been identified with both the stellar structure and the gas dynamics of a forming core. Here we report a candidate core in the process of formation 11 billion years ago, at redshift $z=2.3$. This galaxy, GOODS-N-774, has a stellar mass of 100 billion solar masses, a half-light radius of 1.0 kiloparsecs and a star formation rate of $90_{-20}^{+45}$ solar masses per year. The star-forming gas has a velocity dispersion of $317 \pm 30$ kilometres per second. This is similar to the stellar velocity dispersions of the putative descendants of GOODS$\mathrm{N}-774$, which are compact quiescent galaxies at $z \approx 2$ (refs 8-11) and giant elliptical galaxies in the nearby Universe. Galaxies such as GOODS-N-774 seem to be rare; however, from the star formation rate and size of this galaxy we infer that many star-forming cores may be heavily obscured, and could be missed in optical and near-infrared surveys.

We identified the candidate forming core, GOODS-N-774, using the 3D-HST catalogues in the five CANDELS (Cosmic Assembly NearInfrared Deep Extragalactic Legacy Survey) fields ${ }^{12}$. GOODS-N-774 has a circularized effective radius of $r_{\mathrm{e}}=1.0 \mathrm{kpc}$ from Hubble Space Telescope (HST) F160W $\left(H_{160}\right)$ Wide Field Camera 3 (WFC3) imaging, ${ }^{13}$ a stellar mass of $1.0 \times 10^{11} M_{\odot}$ (refs 12,$14 ; M_{\odot}$, solar mass); rest-frame UVJ colours consistent with a star-forming galaxy; and a Spitzer Multiband Imaging Photometer (MIPS) $24 \mu \mathrm{m}$ flux of $104 \mu \mathrm{Jy}$. Figure 1 shows the stellar mass density profile derived from the observed $H_{160}$ surface brightness profile corrected for the HST point spread function ${ }^{15}$. The surface density profile is strikingly similar to the average profile of massive quiescent galaxies at $z \approx 2$ (red line), and much more concentrated than the average profile of massive star-forming galaxies at that redshift ${ }^{13}$ (light blue).

The near-infrared spectrum of GOODS-N-774 is shown in Fig. 2. The continuum is clearly detected, along with emission lines that we identify as $\mathrm{H} \alpha$ and $[\mathrm{N} \mathrm{II}]$ redshifted to $z=2.300$. The gas velocity dispersion is $\sigma=317 \pm 30 \mathrm{~km} \mathrm{~s}^{-1}$, equivalent to a full-width at half-maximum of $750 \mathrm{~km} \mathrm{~s}^{-1}$. Typically, objects with such large linewidths are mergers or are dominated by active galactic nuclei ${ }^{4}$ (AGNs). If the line emission in GOODS-N-774 is partly or largely due to the presence of an AGN, its velocity dispersion, size and stellar mass measurements would not be reliable.

There is no evidence for the presence of an AGN in GOODS-N-774. It is not detected in the deep Chandra $2 \mathrm{Ms}$ X-ray data in GOODSNorth with an X-ray luminosity upper limit of $L_{\mathrm{X}}<1.2 \times 10^{42} \mathrm{erg} \mathrm{s}^{-1}$. Although an AGN cannot be conclusively ruled out, this upper limit is consistent with the star formation rate of the galaxy. Also, the galaxy has line ratios $[\mathrm{O} \mathrm{III}] /[\mathrm{O} \mathrm{II}]=0.7 \pm 0.5,[\mathrm{O} \mathrm{III}] / \mathrm{H} \beta=1.2 \pm 0.9$ and $[\mathrm{N} \mathrm{II}] /$ $\mathrm{H} \alpha=0.4 \pm 0.1$, indicating that the gas is in a low-ionization state.
Therefore, stellar photoionization and, hence, ultimately, star formation, is the likely origin of the line emission. Finally, the observed infrared spectral energy distribution (SED) requires strong polycyclic aromatic hydrocarbon emission to simultaneously explain the MIPS $24 \mu \mathrm{m}$ and Herschel data (Fig. 3), effectively ruling out the presence of a dominant AGN. We quantified this by fitting composite SEDs with varying AGN

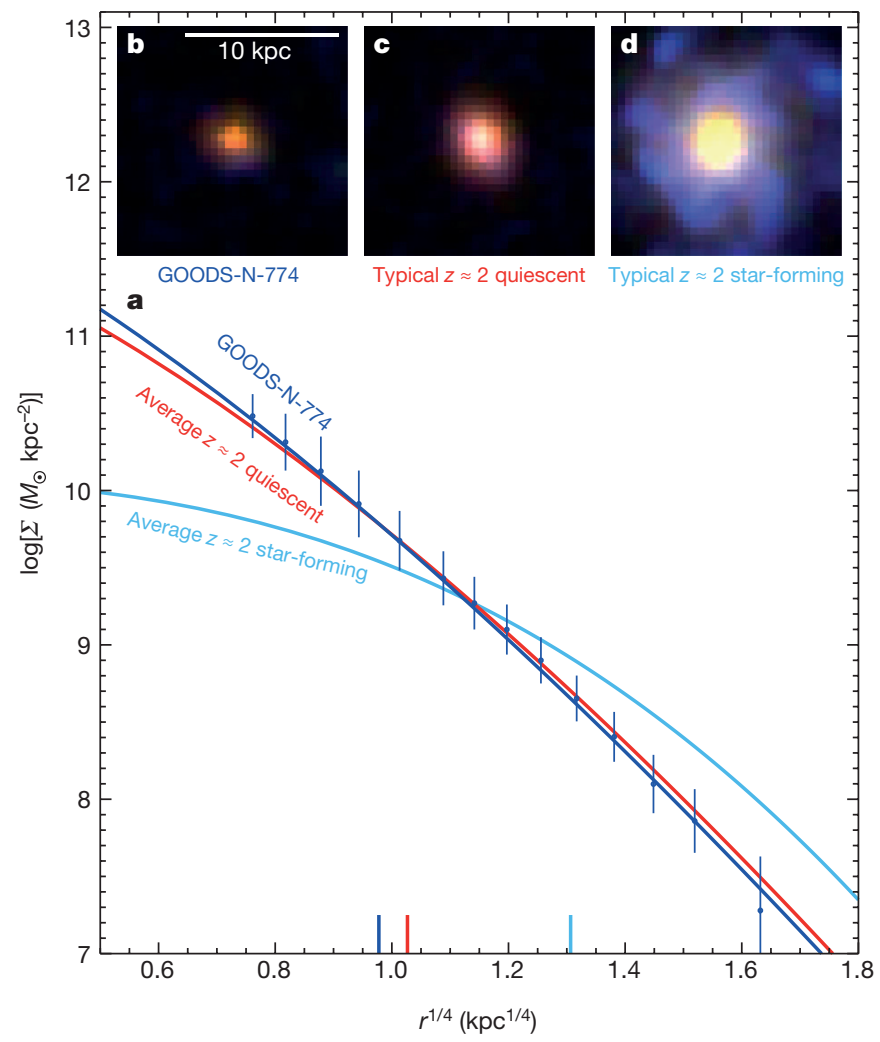

Figure 1 | Structural properties of GOODS-N-774. a, Surface density profile of GOODS-N-774 (blue line), as derived from deep WFC $3 H_{160}$ imaging. Error bars are s.d. The galaxy has a mass of $1.0 \times 10^{11} \mathrm{M}_{\odot}$ and an effective radius of $r_{\mathrm{e}}=1.0 \mathrm{kpc}$. The light blue curve shows the average profile of 67 star-forming galaxies at $1.9<z<2.1$ with $10.9<\log \left(M_{\text {stellar }}\right)<11.2$ (refs 12,13$)$. The red curve shows the average profile of 24 quiescent galaxies with the same mass and redshift selection criteria. b-d, Colour images show GOODS-N-774 (b), a typical quiescent galaxy (c) and a typical star-forming galaxy (d). Vertical bars along the $x$ axis in $\mathbf{a}$ indicate effective radii for the galaxies in $\mathbf{b}-\mathbf{d}$ (colour-coded). The structure of GOODS-N-774 is similar to that of massive quiescent galaxies.

${ }^{1}$ Astronomy Department, Yale University, New Haven, Connecticut 06511, USA. ${ }^{2}$ Leiden Observatory, Leiden University, NL-2300 RA Leiden, The Netherlands. ${ }^{3}$ Space Telescope Science Institute, 3700 San Martin Drive, Baltimore, Maryland 21218, USA. ${ }^{4}$ Max-Planck-Institut für Extraterrestrische Physik, Giessenbackstrasse 1, 85748 Garching, Germany. ${ }^{5}$ Max-Planck-Institut für Astronomie, Königstuhl 17, D-69117 Heidelberg, Germany. ${ }^{6}$ Steward Observatory, University of Arizona, 933 North Cherry Avenue, Tucson, Arizona 85721, USA. ${ }^{7}$ Department of Astronomy, University of Massachusetts, Amherst, Massachusetts 01002, USA. ${ }^{8}$ South African Astronomical Observatory, PO Box 9, Observatory, Cape Town 7935, South Africa. ${ }^{9}$ Astrophysics Science Division, Goddard Space Center, Greenbelt, Maryland 20771, USA. 


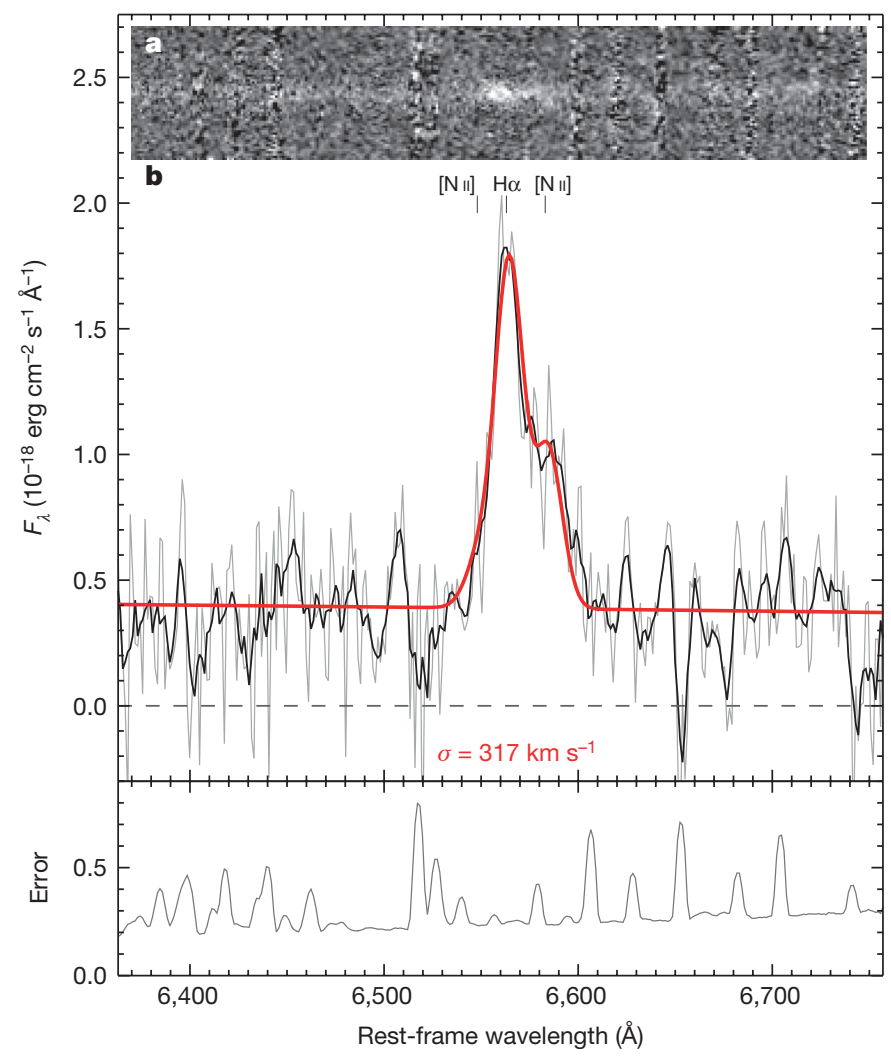

Figure $2 \mid$ Velocity dispersion of GOODS-N-774. Near-infrared spectrum in two dimensions (a) and 1D (b) obtained with NIRSPEC on Keck I. The grey curve is at the original resolution; the black curve shows the spectrum smoothed with a $20 \AA$ boxcar. The best-fit Gaussians to the $\mathrm{H} \alpha \lambda=6,563 \AA$ and [N II] $\lambda=6,548$ and 6,584 $\AA$ emission lines are shown in red. The velocity dispersion is $317 \pm 30 \mathrm{~km} \mathrm{~s}^{-1}$, which is equivalent to an inclination-corrected circular velocity of $V_{\text {rot }} \approx 680 \mathrm{~km} \mathrm{~s}^{-1}$ if the gas is rotating in a disk. The restframe equivalent width of $\mathrm{H} \alpha$ is $66 \pm 8 \AA$ and its luminosity is $(3.4 \pm 0.4) \times 10^{42} \mathrm{erg} \mathrm{s}^{-1}$

contributions ${ }^{16}$. The best fit is obtained for a pure star-forming template with no AGN contribution (Fig. 3).

We infer that the linewidth of GOODS-N-774 is among the highest measured for a normal star-forming galaxy at high redshift (Extended Data Fig. 1). If the gas is in a disk, it is rotating with a velocity of $V_{\text {rot }} \approx$ $550 \mathrm{~km} \mathrm{~s}^{-1}$, or $V_{\text {rot }} \approx 680 \mathrm{~km} \mathrm{~s}^{-1}$ after correcting for inclination. The observed gas velocity dispersion of $317 \mathrm{~km} \mathrm{~s}^{-1}$ is similar to the median stellar velocity dispersion of $304 \mathrm{~km} \mathrm{~s}^{-1}$ in a sample of quiescent galaxies at $z=1.5-2.2$ with median mass $1.9 \times 10^{11} M_{\odot}$ (refs 8-11; Fig. 4).

The inferred dynamical mass is $1.5 \times 10^{11} M_{\odot}$, which is roughly 1.5 times the stellar mass, suggesting a gas fraction of $\lesssim 50 \%$. In Fig. 4, we explicitly compare the dynamical and structural properties of GOODS$\mathrm{N}-774$ with those of galaxies in the Sloan Digital Sky Survey (SDSS) and those of quiescent galaxies at $z \approx 2$. The galaxy has a much smaller size and a higher velocity dispersion than do SDSS galaxies of the same total dynamical mass. Its properties are very similar to those of the samples of quiescent galaxies at $z \approx 2$ that have been compiled over the past few years, and we infer that we have identified an example of star-forming galaxies in this region of parameter space.

The H $\alpha$ luminosity is $(3.4 \pm 0.4) \times 10^{42} \mathrm{erg} \mathrm{s}^{-1}$, which implies a minimum star formation rate (with no reddening correction) of $\sim 16 M_{\odot} \mathrm{yr}^{-1}$ for a Chabrier initial mass function ${ }^{17,18}$. The red colour of the galaxy $\left(R_{606}-H_{160}=4.2 \mathrm{mag}\right)$, and the fact that it is detected with MIPS and Herschel, suggests that the actual, dust-corrected star formation rate is much higher. The $24 \mu \mathrm{m}$ flux alone indicates a star formation rate of $135 M_{\odot} \mathrm{yr}^{-1}$ (ref. 19). Fitting the $24 \mu \mathrm{m}-500 \mu \mathrm{m}$ data with empirical composite star-forming SEDs ${ }^{16}$ or theoretical models ${ }^{20}$ gives slightly

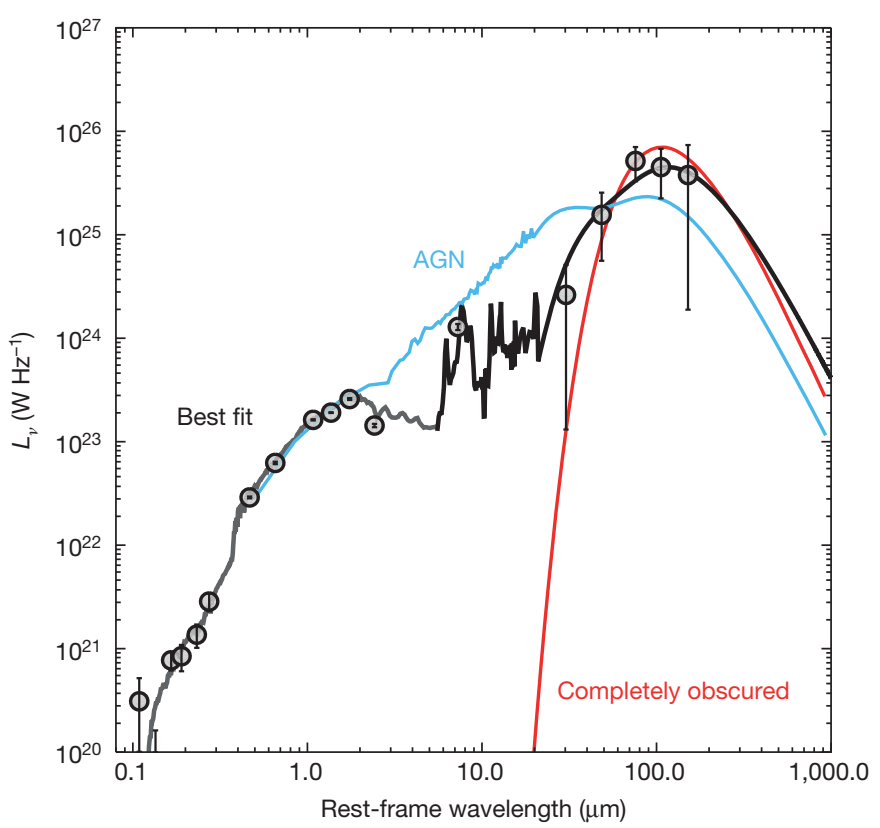

Figure 3 Ultraviolet/far-infrared spectral energy distribution of GOODSN-774. Rest-frame ultraviolet/far-infrared photometry of GOODS-N-774. Error bars are s.d. A stellar population synthesis model fit ${ }^{14}$ to the ultraviolet/ near-infrared SED is shown in grey. The black line shows the composite star-formation/AGN SED ${ }^{16}$ that is the best fit to the mid- and far-infrared data. This best fit has no AGN contribution and implies a star formation rate of $90 M_{\odot} \mathrm{yr}^{-1}$. For reference, the light blue line shows a composite SED with an AGN contribution of $80 \%$. The red curve shows a black body with a size of $1 \mathrm{kpc}$ and a bolometric luminosity of $10^{12}$ solar luminosities.

lower values than do the $24 \mu \mathrm{m}$ data alone, and we infer that the star formation rate is $90_{-20}^{+45} M_{\odot} \mathrm{yr}^{-1}$. This confirms that the star formation is highly obscured, with $\sim 3$ mag of extinction in the direction of the $\mathrm{H} \alpha$ emission and an infrared/ultraviolet luminosity ratio of $\gtrsim 200$.

GOODS-N-774 has a specific star formation rate of $\sim 1 \times 10^{-9} \mathrm{yr}^{-1}$, which places it on the star-forming sequence at $z=2.3$ (ref. 19). If the galaxy had a constant star formation rate leading up to the epoch of observation, then its mass was built up over a period of $\sim 1 \mathrm{Gyr}$ since $z \approx 3.3$. Although short compared with the age of the Universe at $z=$ 2.3 , this build-up phase is $\sim 200$ dynamical times, which is longer than expected from the Kennicutt-Schmidt law ${ }^{21}$. This suggests that the galaxy had a higher star formation rate in the past, or that the star formation rate has been throttled by the rate of gas accretion onto the halo: a galaxy with a stellar mass of $M_{*}=1.0 \times 10^{11} M_{\odot}$ would have a baryonic accretion rate of $\sim(60-120) M_{\odot} \mathrm{yr}^{-1}$ (ref. 22), in good agreement with the observed star formation rate.

The gas in a galaxy such as this, growing by means of rapid star formation in a deep gravitational potential well, should be rapidly enriched with metals, and we would thus expect it to exhibit a high gas-phase metallicity. This is consistent with what we observe: the galaxy has [N II]/ $\mathrm{H} \alpha=0.4 \pm 0.1$, which implies a high metallicity $(12+\log (\mathrm{O} / \mathrm{H}) \approx 9.05$, although the conversion ${ }^{23}$ is somewhat uncertain). After the star formation phase, the gas is probably heated, expelled or both ${ }^{2,22}$. The quiescent core that remains will then probably evolve into a giant elliptical galaxy ${ }^{2,3}$ with a central stellar metallicity that is similar to the gas-phase metallicity of the star-forming core at high redshift ${ }^{24}$.

Galaxies such as GOODS-N-774 are rare. Candidate compact starforming galaxies with less extreme properties have been identified in fairly large numbers ${ }^{5,6}$, but in the $\sim 900 \mathrm{arcmin}^{2}$ of the five 3D-HST CANDELS fields there are only three objects at $2<z<3$ with $24 \mu \mathrm{m}$ fluxes of $\geq 100 \mu \mathrm{Jy}$, high central mass densities $\left(\log \left(M / M_{\odot}\right)_{r<1 \mathrm{kpc}} \geq\right.$ 10.5; ref. 7) and concentrated stellar distributions $\left(r_{\mathrm{e}} \leq 1 \mathrm{kpc}\right)$. We observed all three galaxies with Keck I, and GOODS-N-774 is the only 

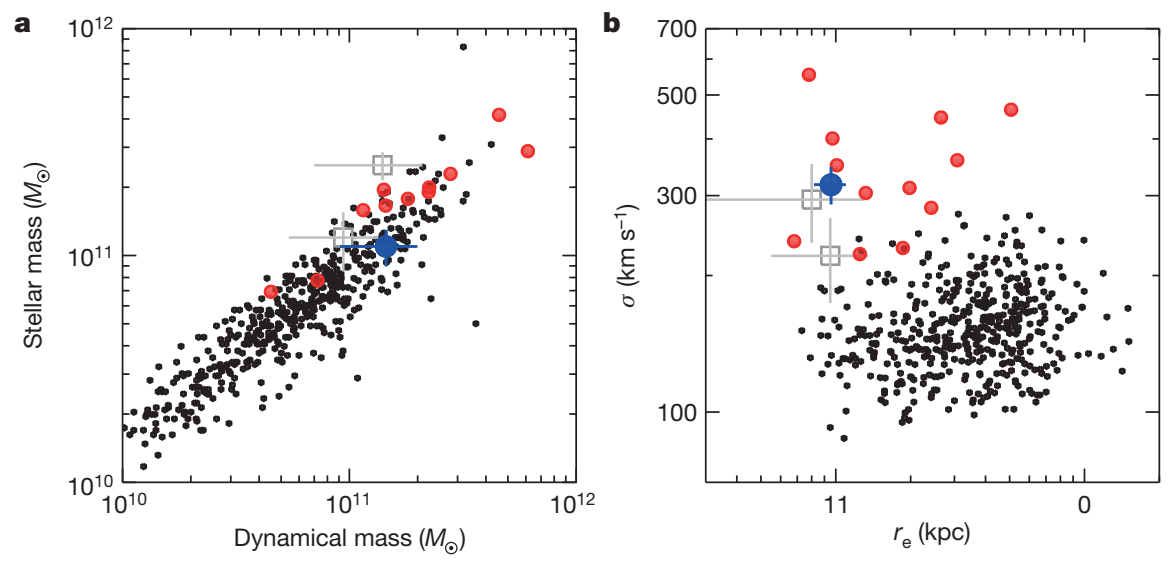

Figure $4 \mid$ Properties of GOODS-N-774 compared with quiescent galaxies. Comparisons of the size, mass and gas dynamics of GOODS-N774 (blue symbols) to the sizes, masses and stellar dynamics of galaxies in the SDSS (black) and massive quiescent galaxies at $z \approx 2(\mathrm{red})^{8-11}$. GOODS-N-774 has properties that are similar to previously studied massive quiescent galaxies at $z \approx 2$ and is substantially offset from nearby galaxies. $\mathrm{CO}$ dynamics and $\mathrm{CO}$ sizes of two compact SMGs from ref. 13 (HDF 76 and N2850.2) are shown in grey. Error bars are s.d.
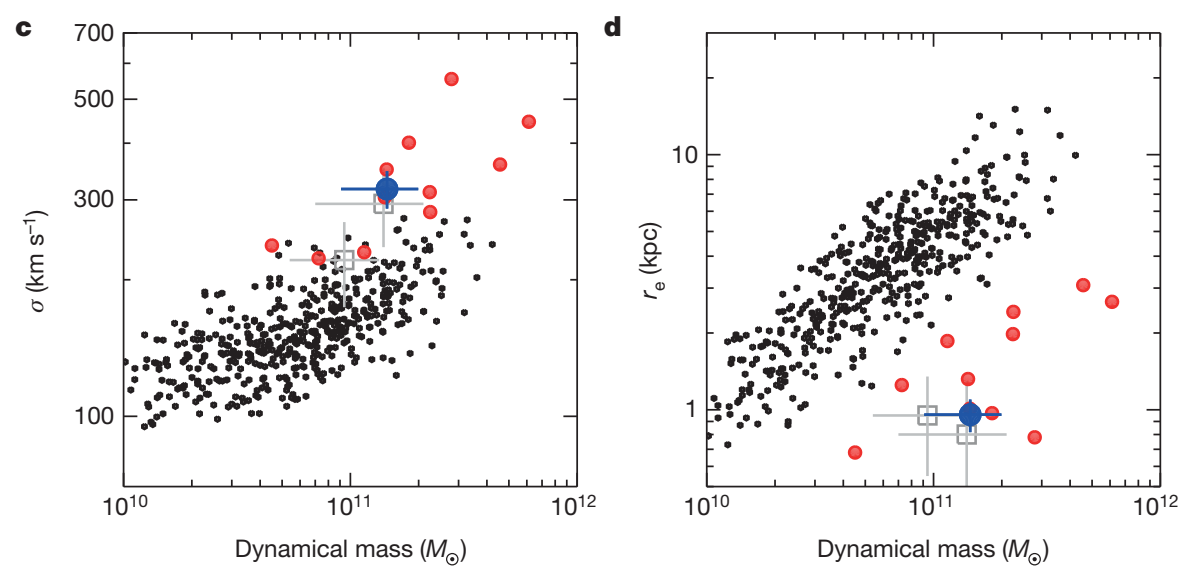

confirmed candidate: GOODS-S-5981 ${ }^{6}$ has a narrow linewidth, whereas COSMOS-8388 is difficult to interpret because it has an AGN. The number density we infer is $\sim 10^{-6} \mathrm{Mpc}^{-3}$ (including all three candidates), compared with $\sim 10^{-4} \mathrm{Mpc}^{-3}$ for the overall population of galaxies with dense cores at $z \approx 2$ (ref. 3 ).

This mismatch could imply that the lifetime of the compact starforming phase is very short, as has been suggested previously on the basis of similar number density arguments ${ }^{4}$. It may be that we are witnessing the aftermath of the contraction of a gravitationally unstable star-forming disk $^{25}$ or of a merger of large star-forming galaxies ${ }^{4}$. However, neither tidal features nor extended wings are apparent in the surface density distribution.

It is perhaps more likely that the lifetime of the compact star-forming phase is relatively long and that we are missing many star-forming compact galaxies in present surveys. From the compact morphology and high star formation rate, we infer a high gas column density for this object ${ }^{21}: N_{\mathrm{H}}=2.6 \times 10^{23} \mathrm{~cm}^{-2}$. This gas column density is nearly an order of magnitude higher than in an average ultraviolet-selected starforming galaxy at the same cosmic epoch ${ }^{26}$, and 2.5 orders of magnitude higher than in the disk of a typical galaxy in the local Universe ${ }^{21}$. This high column density of gas in conjunction with the abundance of metals implies ${ }^{27}$ a very high extinction: $A_{\mathrm{V}} \gtrsim 100$ mag for a screen (V, visual band), and $A_{\mathrm{V}} \gtrsim 6$ mag if the dust and the stars are mixed. The amount of extinction is driven by the dust column density, not the dust mass, meaning that at fixed dust mass, a compact galaxy will be more obscured than a large galaxy. The detection of rest-frame optical flux, and of $\mathrm{H} \alpha$ emission, is inconsistent with such high values for extinction. The dust distribution is probably non-uniform, and it may be that, for GOODS-N-774, we are looking along a relatively unobscured line of sight.

More typical star-forming cores could be entirely obscured ${ }^{27,28}$, and begin to resemble black bodies with temperatures of $\sim 30 \mathrm{~K}$ (red curve in Fig. 3; calculated using a radius of $1 \mathrm{kpc}$ and a bolometric luminosity of $10^{12}$ solar luminosities). It may be possible to select such obscured progenitors at long wavelengths, near the peak of the redshifted dust emission. It has been demonstrated that redshifts, sizes and velocity widths of infrared-luminous galaxies can be measured from $\mathrm{CO}$ emission. In fact, the closest analogues to GOODS-N-774 are the two submillimetreselected galaxies (SMGs) HDF 76 and N2850.2 (Fig. 4), which have high linewidths and small sizes in the CO line ${ }^{4}$. It will be interesting to determine whether the stellar distributions of these galaxies are similar to the gas distribution, or whether these are dense star-forming regions inside larger galaxies.

Longer-wavelength studies of large, unbiased samples can show whether GOODS-N-774 is an example of a parent population of compact starforming galaxies that are heavily obscured ${ }^{4}$. There may also be multiple paths to a compact, quiescent galaxy: some (such as HDF 76 and $\mathrm{N} 2850.2^{4}$ ) may form most of their stars in mergers with star formation rates of $\gtrsim 1,000 M_{\odot} \mathrm{yr}^{-1}$, whereas others (such as GOODS-N-774) may grow relatively slowly in an obscured, accretion-throttled mode. Whatever the dominant mode turns out to be, because the stars in dense cores account for $10-20 \%$ of the total $z \approx 2$ stellar mass density ${ }^{3}$, star-forming cores should account for a significant fraction of all star formation in the high-redshift Universe.

Very recently, evidence supporting our conclusions has been posted online $e^{29}$.

Online Content Methods, along with any additional Extended Data display items and Source Data, are available in the online version of the paper; references unique to these sections appear only in the online paper.

Received 4 May; accepted 19 June 2014 Published online 27 August 2014.

1. Daddi, E. et al. Passively evolving early-type galaxies at $1.4 \lesssim z \lesssim 2.5$ in the Hubble Ultra Deep Field. Astrophys. J. 626, 680-697 (2005).

2. Oser, L., Ostriker, J. P., Naab, T., Johansson, P. H. \& Burkert, A. The two phases of galaxy formation. Astrophys. J. 725, 2312-2323 (2010).

3. van Dokkum, P. et al. Dense cores in galaxies out to $z=2.5$ in SDSS, UItraVISTA, and the five 3D-HST/CANDELS fields: number density, evolution, and the 
apparent need for efficient cooling at high redshift. Astrophys. J. (submitted); preprint at http://arxiv.org/abs/1404.4874 (2014).

4. Tacconi, L. et al. Submillimeter galaxies at $z \sim 2$ : evidence for major mergers and constraints on lifetimes, IMF, and CO- $\mathrm{H}_{2}$ conversion factor. Astrophys. J. 680 , 246-262 (2008).

5. Toft, S. et al. Submillimeter galaxies as progenitors of compact quiescent galaxies. Astrophys. J. 782, 68 (2014)

6. Barro, G. et al. CANDELS+3D-HST: compact SFGs at $z \sim 2-3$, the progenitors of the first quiescent galaxies. Preprint at http://arxiv.org/abs/1311.5559 (2013).

7. Williams, C. C. et al. The progenitors of the compact early-type galaxies at high redshift. Astrophys. J. 780, 1 (2014).

8. Bezanson, R., van Dokkum, P., van de Sande, J., Franx, M. \& Kriek, M. Massive and newly dead: discovery of a significant population of galaxies with high-velocity dispersions and strong Balmer lines at $z \sim 1.5$ from deep Keck spectra and HST/ WFC3 imaging. Astrophys. J. 764, L8 (2013).

9. van Dokkum, P. G., Kriek, M. \& Franx, M. A high stellar velocity dispersion for a compact massive galaxy at redshift $z=2.186$. Nature 460, 717-719 (2009).

10. van de Sande, J. etal. Stellar kinematics of $z \sim 2$ galaxies and the inside-out growth of quiescent galaxies. Astrophys. J. 771, 85 (2013)

11. Belli, S., Newman, A. B. \& Ellis, R. S. Velocity dispersions and dynamical masses for a large sample of quiescent galaxies at $z>1$ : improved measures of the growth in mass and size. Astrophys. J. 783, 117 (2014).

12. Skelton, R. E. et al. 3D-HST WFC3-selected photometric catalogs in the five CANDELS/3D-HST fields: photometry, photometric redshifts and stellar masses. Preprint at http://arxiv.org/abs/1403.3689 (2014).

13. van der Wel, A. et al. 3D-HST+CANDELS: the evolution of the galaxy size-mass distribution since $z=3$. Astrophys. J. 788, 28 (2014)

14. Kriek, M. etal. An ultra-deep near-infrared spectrum of a compact quiescent galaxy at $z=2.2$. Astrophys. J. 700, 221-231 (2009)

15. Szomoru, D. et al. Confirmation of the compactness of a $z=1.91$ quiescent galaxy with Hubble Space Telescope's Wide Field Camera 3. Astrophys. J. 714, L244-L248 (2010).

16. Kirkpatrick, A. et al. GOODS-Herschel: impact of active galactic nuclei and star formation activity on infrared spectral energy distributions at high redshift. Astrophys. J. 759, 139 (2012)

17. Kennicutt, R. C. Jr. Star formation in galaxies along the Hubble sequence. Annu. Rev. Astron. Astrophys. 36, 189-232 (1998)

18. Chabrier, G. Galactic stellar and substellar initial mass function. Publ. Astron. Soc. Pacif. 115, 763-795 (2003).
19. Wuyts, S etal Galaxy structure and mode of star formation in the SFR-mass plane from $z \sim 2.5$ to $z \sim 0.1$. Astrophys. J. 742, 96 (2011)

20. da Cunha, E., Charlot, S. \& Elbaz, D. A simple model to interpret the ultraviolet, optical and infrared emission from galaxies. Mon. Not. R. Astron. Soc. $\mathbf{3 8 8}$, 1595-1617 (2008).

21. Kennicutt, R. C. Jr. The global Schmidt law in star-forming galaxies. Astrophys. J. 498, 541 (1998).

22. Dekel, A. et al. Toy models for galaxy formation versus simulations. Mon. Not. R. Astron. Soc. 435, 999-1019 (2013).

23. Maiolino, R. et al. AMAZE. I. The evolution of the mass-metallicity relation at $z>3$. Astron. Astrophys. 488, 463-479 (2008).

24. Leja, J. et al. Exploring the chemical link between local ellipticals and their highredshift progenitors. Astrophys. J. 778, L24 (2013)

25. Dekel, A. \& Burkert, A. Wet disk contraction to galactic blue nuggets and quenching to red nuggets. Mon. Not. R. Astron. Soc. 438, 1870 (2014).

26. Erb, D. K. et al. $\alpha$ observations of a large sample of galaxies at $z \sim 2$ : implications for star formation in high-redshift galaxies. Astrophys. J. 647, 128-139 (2006).

27. Gilli, R. et al. ALMA reveals a warm and compact starburst around a heavily obscured supermassive black hole at $z=4.75$. Astron. Astrophys. 562, A67 (2014).

28. Wang, W.-H., Barger, A. J. \& Cowie, L. L. A Ks and IRAC selection of high-redshift extremely red objects. Astrophys. J. 744, 155 (2012).

29. Barro, G. et al. Keck-I MOSFIRE spectroscopy of compact star-forming galaxies at $z \gtrsim 2$ : high velocity dispersions in progenitors of compact quiescent galaxies. Preprint at http://arxiv.org/abs/1405.7042 (2014).

Acknowledgements Support from STScl grant GO-1277 is acknowledged.

Author Contributions E.N. obtained the data, led the analysis and the interpretation, and wrote the manuscript. P.v.D. contributed to the analysis and the interpretation. M.F. contributed to the interpretation. I.M. reduced the WFC3 imaging. G.B. and I.M. reduced the grism spectroscopy. K.W. and R.S. led the photometric analysis. All authors commented on the manuscript.

Author Information Reprints and permissions information is available at www.nature.com/reprints. The authors declare no competing financial interests. Readers are welcome to comment on the online version of the paper. Correspondence and requests for materials should be addressed to E.N. (erica.nelson@yale.edu). 


\section{METHODS}

Spectral energy distribution. The candidate forming core was found using the 3D-HST catalogues in the five CANDELS fields. CANDELS is a 902-orbit Hubble Space Telescope program that provides space-based optical and near-infrared imaging across $\sim 900 \operatorname{arcmin}^{2}$ (refs 30,31). Aperture photometry was performed to produce publicly available photometric catalogues and to derive stellar masses ${ }^{12,14}$. Spitzer MIPS $24 \mu \mathrm{m}$ fluxes were determined using the same methodology as in ref. 32. The derived fluxes are consistent with the public catalogue of ref. 33. Using the $24 \mu \mathrm{m}$ data as position priors, we measure the $100-500 \mu \mathrm{m}$ fluxes from the ultra-deep Herschel imaging in GOODS-North ${ }^{34}$. In sum, the rest-frame ultraviolet-optical data come from HST/ACS, HST/WFC3 and ground-based optical telescopes; the rest-frame near-infrared data are from Spitzer/IRAC; the mid-infrared point is from Spitzer/ MIPS; and the far-infrared data are from Herschel/PACS and SPIRE.

Keck spectroscopy. We observed GOODS-N-774 with the near infrared spectrograph (NIRSPEC) on the Keck I telescope in the K band, on 11 January 2014. The total integration time was $6,000 \mathrm{~s}$. We used the low-dispersion mode with a slit width of $0.7^{\prime \prime}$, giving a spectral resolution of $\sigma_{\text {instr }}=6.1 \AA$ in the rest frame. We fitted a Gaussian to the $\mathrm{H} \alpha \lambda=6,563 \AA$ and [N II] $\lambda=6,548$ and 6,584 $\AA$ emission lines simultaneously and corrected for the instrumental resolution. The uncertainty in the derived properties was determined by refitting the model with empirical realizations of the noise.

HST grism spectroscopy. A WFC3/G141 grism spectrum of the object was obtained as part of the 3D-HST survey ${ }^{35}$. 3D-HST is a near-infrared slitless spectroscopic Treasury programme. We examined the grism spectrum after measuring a secure redshift from the Keck/NIRSPEC spectrum. The redshifted [O II], $\mathrm{H} \beta$ and [O III] lines are detected with a significance of $1.5 \sigma-2.5 \sigma$. GOODS-N-774 has optical emission line ratios $[\mathrm{O} \mathrm{III}] / \mathrm{H} \beta=1.2 \pm 0.9$ and $[\mathrm{N} \mathrm{II}] / \mathrm{H} \alpha=0.4 \pm 0.1$, suggesting a level of gas excitation that is slightly higher than the locus of star-forming galaxies in the local Universe ${ }^{36}$ but at the low end for star-forming galaxies at $z \approx 2$ (ref. 37) in the diagnostic BPT diagram.

X-ray constraints. GOODS-N-774 is in the Chandra Deep Field North, which has been observed for a total of $\sim 2 \mathrm{Ms}$ with the Chandra X-ray satellite. The exposure time at the location of GOODS-N-774 is $1.22 \mathrm{Ms}$. The galaxy is not in the publicly available point-source catalogue of this field ${ }^{38}$. There are seven counts in a $3^{\prime \prime}$ aperture centred on the object location in the full-band $(0.5-8 \mathrm{keV}) \mathrm{X}$-ray image, fully consistent with the counts in random apertures in regions with the same exposure time. Using the s.d. of the counts in random apertures, we derive a 2 s.d. upper limit of six counts for the $\mathrm{X}$-ray flux of GOODS-N-774. Using PIMMS v4.6b, we derive a rest-frame 2-10 keV flux limit of $F_{\mathrm{X}}<2.9 \times 10^{-17} \mathrm{erg} \mathrm{s}^{-1} \mathrm{~cm}^{-2}$, corresponding to a luminosity of $L_{\mathrm{X}}<1.2 \times 10^{42} \mathrm{erg} \mathrm{s}^{-1}$. We conclude that there is no evidence for an AGN in GOODS-N-774. The upper limit is consistent with the star formation rate of the galaxy $y^{39}$.

Gas column density. We derive the gas surface density using the KennicuttSchmidt law ${ }^{21}$ :

$$
\Sigma_{\mathrm{SFR}}=(2.5 \pm 0.7) \times 10^{-4}\left(\frac{\sum_{\mathrm{gas}}}{1 M_{\odot} \mathrm{pc}^{-2}}\right)^{1.4 \pm 0.15} M_{\odot} \mathrm{yr}^{-1} \mathrm{kpc}^{-2}
$$

Dynamical mass. We define dynamical mass as $M_{\mathrm{dyn}}=k(n) \sigma^{2} r_{\mathrm{e}} / G$, with the constant $k(n)$ depending on the Sérsic index: $k(n)=8.87-0.831 n+0.0241 n^{2}$ (ref. 40). GOODS-N-774 has a Sérsic index of $n=2.9$; the comparison samples of compact quiescent galaxies at $z \approx 2$ (refs 1, 41-45) and SDSS galaxies with $0.058<z<0.060$ have median indices of $n=3.2$ and $n=4.1$, respectively.

30. Grogin, N. et al. CANDELS: the Cosmic Assembly Near-infrared Deep Extragalactic Legacy Survey. Astrophys. J. Suppl. Ser. 197, 35 (2011).

31. Koekemoer, A. et al. CANDELS: the Cosmic Assembly Near-infrared Deep Extragalactic Legacy Survey - the Hubble Space Telescope observations, imaging data products and mosaics. Astrophys. J. Suppl. Ser. 197, $36(2011)$

32. Whitaker, K. et al. The NEWFIRM medium-band survey: photometric catalogs, redshifts, and the bimodal color distribution of galaxies out to $z \sim 3$. Astrophys. J. 735, 86 (2011).

33. Lutz, D. et al. PACS Evolutionary Probe (PEP) - a Herschel key program. Astron. Astrophys. 532, A90 (2011)

34. Elbaz, D. et al. GOODS-Herschel: an infrared main sequence for star-forming galaxies. Astron. Astrophys. 533, A119 (2011).

35. Brammer, G. B. et al. 3D-HST: a wide-field grism spectroscopic survey with the Hubble Space Telescope. Astrophys. J. 200, 13 (2012).

36. Tremonti, C. et al. The origin of the mass-metallicity relation: insights from 53,000 star-forming galaxies in the Sloan Digital Sky Survey. Astrophys. J. 613, 898 (2004)

37. Steidel, C. et al. Strong nebular line ratios in the spectra of $z \sim 2-3$ star-forming galaxies: first results from KBSS-MOSFIRE. Astrophys. J. (submitted); preprint at http://arxiv.org/abs/1405.5473 (2014).

38. Alexander, D. et al. The Chandra Deep Field North Survey. XIII. 2 Ms point-source catalogs. Astron. J. 126, 539 (2003)

39. Grimm, H.-J, Gilfanov, M. \& Sunyaev, R. High-mass X-ray binaries as a star formation rate indicator in distant galaxies. Mon. Not. R. Astron. Soc. 339, 793 (2003).

40. Bertin, G., Ciotti, L. \& Del Principe, M. Weak homology of elliptical galaxies. Astron Astrophys. 386, 149-168 (2002).

41. Trujillo, I. et al. The size evolution of galaxies since $z \sim 3$ : combining SDSS, GEMS, and FIRES. Astrophys. J. 650, 18-41 (2006).

42. Toft, S. et al. Hubble Space Telescope and Spitzer imaging of red and blue galaxies at $z \sim 2.5$ : a correlation between size and star formation activity from compact quiescent galaxies to extended star-forming galaxies. Astrophys. J. 671, 285-302 (2007).

43. van Dokkum, P. G. et al. Confirmation of the remarkable compactness of massive quiescent galaxies at $z \sim 2.3$ : early-type galaxies did not form in a simple monolithic collapse. Astrophys. J. 677, L5-L8 (2008).

44. Cimatti, A. et al. GMASS ultradeep spectroscopy of galaxies at $z \sim 2$. II. Superdense passive galaxies: how did they form and evolve? Astron. Astrophys. 482, 21-42 (2008).

45. Newman, A. B., Ellis, R. S., Treu, T. \& Bundy, K. Keck spectroscopy of $z>1$ field spheroidals: dynamical constraints on the growth rate of red "nuggets". Astrophys. J. 717, L103-L107 (2010).

46. Förster Schreiber, N. M. et al. The SINS survey: SINFONI integral field spectroscopy of $z \sim 2$ star-forming galaxies. Astrophys. J. 706, 1364-1428 (2009). 


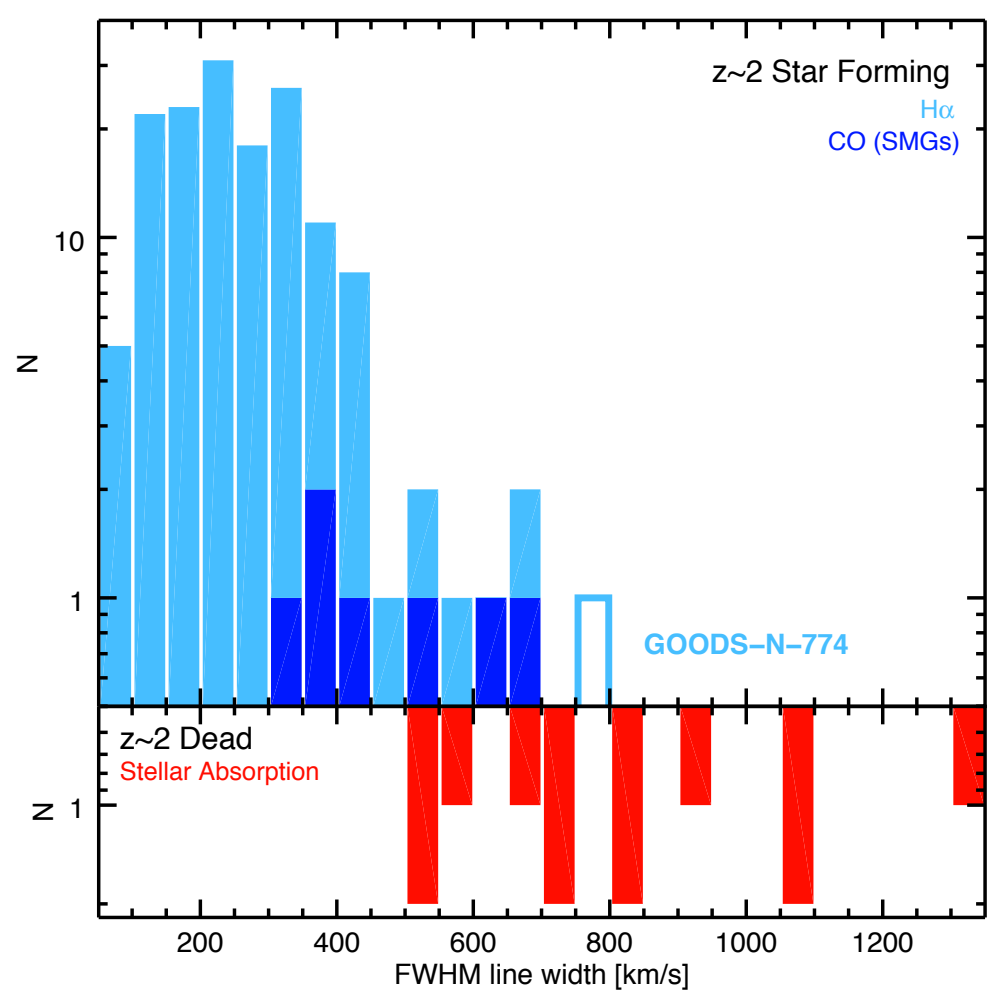

Extended Data Figure $1 \mid$ Linewidths of $z \approx 2$ star-forming and quiescent galaxies. The linewidth of GOODS-N-774 (open box) is among the

dispersion is similar to the median stellar velocity dispersion of $304 \mathrm{~km} \mathrm{~s}^{-1}$ highest measured for a normal star-forming galaxy at high redshift in $\mathrm{H} \alpha$ in a sample of quiescent galaxies at $z=1.5-2.2$ with median mass of emission $^{26,46}$ (light blue) or CO emission ${ }^{4}$ (SMGs; dark blue). The gas velocity $1.9 \times 10^{11} M_{\odot}($ refs $8-11 ;$ red $)$. 